\title{
Using the zebrafish model for Alzheimer's disease research
}

\section{Morgan Newman*, Esmaeil Ebrahimie and Michael Lardelli}

School of Molecular and Biomedical Science, University of Adelaide, SA, Australia

\section{Edited by: \\ Shin Murakami, Touro University \\ California, USA}

\section{Reviewed by:}

Fred Van Leeuwen, Maastricht University, Netherlands

Ruishan Wang, University of

Tennessee Health Science Center, USA

\section{${ }^{*}$ Correspondence:}

Morgan Newman, School of

Molecular and Biomedical Science, University of Adelaide, SA 5005,

Australia

e-mail:morgan.newman@adelaide. edu.au
Rodent models have been extensively used to investigate the cause and mechanisms behind Alzheimer's disease. Despite many years of intensive research using these models we still lack a detailed understanding of the molecular events that lead to neurodegeneration. Although zebrafish lack the complexity of advanced cognitive behaviors evident in rodent models they have proven to be a very informative model for the study of human diseases. In this review we give an overview of how the zebrafish has been used to study Alzheimer's disease. Zebrafish possess genes orthologous to those mutated in familial Alzheimer's disease and research using zebrafish has revealed unique characteristics of these genes that have been difficult to observe in rodent models. The zebrafish is becoming an increasingly popular model for the investigation of Alzheimer's disease and will complement studies using other models to help complete our understanding of this disease.

Keywords: zebrafish, Alzheimer disease, presenilins, gamma-secretase, amyloid beta-protein precursor, MAPT

\section{ALZHEIMER'S DISEASE}

Alzheimer's disease (AD) is the most prevalent form of dementia. It was estimated in 2010 that 36 million people were living with $\mathrm{AD}$ and this figure is predicted to increase to 66 million by 2030 (World Alzheimer report's, 2010). The main clinical feature of AD is progressive memory loss. However, $\mathrm{AD}$ can also be characterized by the impairment of speech and motor ability, depression, delusions, hallucinations, and aggressive behavior (Voisin and Vellas, 2009). Despite these recognizable behavioral differences it is actually difficult during the early stages of the disease to correctly diagnose someone as having $\mathrm{AD}$ (Blennow et al., 2006). There is significant neuronal loss in several brain regions in AD patients (Regeur et al., 1994; West et al., 1994). This is usually accompanied by extracellular aggregates of the amyloid-beta peptide and intracellular aggregates of the tau protein called neurofibrillary tangles (NFTs).

Alzheimer's disease can be classed as familial (FAD, usually with onset $<65$ years) or sporadic (SAD, onset $>65$ years). We have acquired most of our knowledge on the pathogenesis of $\mathrm{AD}$ through studies of FAD mutations. FAD is attributed to mutations in three genes, PRESENILIN 1 (PSEN1), PRESENILIN 2 (PSEN2), and the AMYLOID BETA A4 PRECURSOR PROTEIN $(A P P)$. The PSEN proteins are essential for $\gamma$-secretase activity that cleaves transmembrane domain proteins within lipid bilayers (reviewed in McCarthy et al., 2009). The PSEN proteins and four other proteins comprise $\gamma$-secretase complexes. PSEN ( 1 or 2), nicastrin (NCT), anterior pharynx defective 1 (APH1a or APH1b), and presenilin enhancer 2 (PSENEN) form active $\gamma$-secretase complexes in cellular membranes. These complexes are responsible for the cleavage of a number of single-pass transmembrane proteins such as APP and NOTCH. APP is initially cleaved by $\alpha$ - or $\beta$-secretases to release APP $\alpha$ or APP $\beta$ fragments, respectively. The subsequent cleavage of APP by $\gamma$-secretase after $\beta$-secretase cleavage liberates amyloid- $\beta$ (A $\beta$ ) peptides of various lengths. The longer $A \beta-42$ peptide is prone to aggregation and is suggested to form toxic oligomers and fibrils that eventually deposit as amyloid plaques in the brain. Deposits of plaques and NFTs are common occurrences in the brains of those with FAD.

The common, late onset form of $\mathrm{AD}$ (accounting for $>90 \%$ of cases) occurs sporadically (Blennow et al., 2006). Sporadic $\mathrm{AD}(\mathrm{SAD})$ has a complex etiology and is associated with many risk factors including old-age and possession of the $\varepsilon 4$ allele of the APOLIPOPROTEIN E (APOE) gene. Human genome wide association studies have revealed a number of possible loci associated with risk for SAD [reviewed by Rademakers and Rovelet-Lecrux, 2009). There are numerous other risk factors associated with an individual's lifestyle that can also increase the likelihood of developing AD. These overlap considerably with the risk factors for cardiovascular disease (Kotze and van Rensburg, 2012) including hypertension, hypercholesterolemia and obesity (Martins et al., 2006). The molecular and cellular triggers for the onset of SAD are yet to be completely resolved, however, there have been many hypotheses suggested to explain how $\mathrm{AD}$ is initiated. Of the many hypotheses that have been postulated over the years, some that are gaining momentum attribute AD to vasculature dysfunction (Stone, 2008; Marchesi, 2011), oxidative stress (Nunomura et al., 2001), mitochondrial dysfunction (Selfridge etal., 2012) or hypoxia (Oresic etal., 2011).

The common observation of amyloid plaques in $\mathrm{AD}$ brains led to the formulation of the amyloid hypothesis in 1992 (Hardy and Higgins, 1992) that continues to dominate research thinking as a unifying hypothesis for FAD and SAD. The hypothesis suggests that toxic oligomerization of $A \beta$ peptides is the initiating factor that triggers a cascade of subsequent cellular abnormalities such as inflammation and oxidative stress (Verdile et al., 2004). These secondary phenomena ultimately lead to neuronal dysfunction, degeneration and death. Although still widely accepted, doubts have arisen regarding its validity following several failed 
clinical trials of drugs intended to reduce $A \beta$ levels (refer to www.alzforum.org regarding information on $\mathrm{AD}$ drugs in clinical trials).

Rodent models of AD have been exploited extensively and have given considerable insight into this disease. However, we still do not understand the events that trigger the neurodegeneration evident in AD. Research using the zebrafish model has revealed particular characteristics of the various genes implicated in AD that have been difficult to observe in other animal models (Liao et al., 2012; Newman et al., 2012). This review will focus on how the zebrafish has been used to study the genes and various cellular pathways implicated in FAD and SAD pathogenesis and will conclude by examining the limitations of the zebrafish and what the future may hold for use of this model in AD research.

\section{THE ZEBRAFISH MODEL}

The zebrafish are a small, hardy freshwater fish native to India and often kept in home aquaria. They were originally used as a model organism for the study of vertebrate development. However, over the last decade the zebrafish model has become increasingly employed for investigating a wide variety of human diseases (Lieschke and Currie, 2007). The zebrafish has a number of characteristics that make it a versatile animal model. Although they lack the advanced cognitive behaviors evident in rodent models, their transparent embryos, rapid development ex utero and large reproductive capacity $(100+$ embryos per spawning) provide obvious advantages over mammalian models. Furthermore, multiple genes can be manipulated simply and effectively in the zebrafish at physiologically relevant levels (Newman et al., 2012), which cannot be currently achieved in rodent models. While, in general, rodents more closely model human physiology than fish, zebrafish nevertheless are vertebrates and so are more relevant to understanding human biology than invertebrate models as Drosophila melanogaster and Caenorhabditis elegans. Zebrafish embryos are particularly manipulable due to their large size, ready availability and the ability to exploit changes in their development for assay of particular gene activities (Nornes et al., 2009). Thus, zebrafish embryos can often present a felicitous vertebrate system in which to examine the molecular and cellular functions of genes implicated in AD.

The zebrafish genome is extensively annotated. The zebrafish (teleost-bony fish) evolutionary lineage separated from the human (tetrapod) lineage approximately 450 million years ago (Kumar and Hedges, 1998). Teleosts appear to have undergone an additional round of genome duplication since their separation from the tetrapod lineage followed by loss of many of the duplicated genes (Catchen et al., 2011). However, in most cases, zebrafish genes can be identified that are clear orthologs of human genes. Zebrafish possess genes orthologous to the human genes that are thought to play essential roles in AD. The psen1 (Leimer et al., 1999) and psen 2 (Groth et al., 2002) genes are orthologs of human PSEN1 and PSEN2, respectively, while the appa and $a p p b$ genes are "co-orthologs" of human APP (Musa et al., 2001). Zebrafish hold orthologous genes for the components of the gamma-secretase complex, PSENEN (psenen; Francis et al., 2002; Campbell et al., 2006), NCTN (ncstn; Strausberg et al., 2002) and APH1b (aph1b;
Francis et al., 2002). While orthologs of $\beta$-secretase (BACE1 and $B A C E 2)$ have also recently been identified in zebrafish: bace1 (Moussavi Nik et al., 2012) and bace2 (van Bebber et al., 2013). The microtubule-associated protein tau (MAPT) gene in humans encodes the tau protein and our laboratory identified co-orthologs of this gene in zebrafish, mapta, and maptb (Chen et al., 2009). Zebrafish also possess co-orthologs of APOE: apoea and apoeb (Babin et al., 1997; Woods et al., 2005). Genes arising by duplication can have overlapping functions. This can be disadvantageous when analyzing gene function as particular loss-of-function phenotypes may be obscured unless the function of both duplicate genes is blocked. Alternatively, duplicate genes can have partially non-overlapping functions and this can facilitate functional analysis as loss-of-function phenotypes may be restricted to particular cells or tissues.

Zebrafish are an advantageous model for genetic and molecular studies. Zebrafish embryos are genetically malleable by injection of morpholino antisense oligonucleotides, mRNA, transgenes and more recently by genome engineering systems (reviewed in Hwang et al., 2013; Schmid and Haass, 2013; Hisano et al., 2014). These technologies can make both subtle and drastic changes in gene expression with the effects observed in the developing transparent embryos. Morpholinos are designed to bind to particular sites in transcripts from a gene of interest. Binding of a morpholino can either block mRNA translation (knockdown) or interfere with correct splicing of exons (e.g., Draper et al., 2001; Nornes et al., 2008; Berger et al., 2011). Injection of sense mRNA can allow overexpression of a particular gene of interest. The effects of morpholino and mRNA injection generally only persist during embryogenesis (2-3 days post-fertilization). Transgenic zebrafish can be generated using efficient vectors such as the Tol 2 transposase system (Kawakami et al., 2000) to insert genes under the control of tissue specific promoters. Conditionally expressed transgenics can also be generated using the Cre/loxP (Hans et al., 2009) and GAL4-UAS (Halpern et al., 2008) systems for gene function analysis at particular time points. The absence of technology available to generate targeted mutations in the zebrafish genome was previously a disadvantage of the zebrafish model relative to rodents. In recent times however, zinc finger nucleases (ZFNs), transcription activator-like effector nucleases (TALENS) and the type II prokaryotic CRISPR (clustered regularly interspaced short palindromic repeats)/Cas systems have been developed for targeted modification of gene sequences in the zebrafish genome (Hwang et al., 2013; Schmid and Haass, 2013).

A search of the available scientific literature in the PubMed database using the term "zebrafish + alzheimer's disease" revealed 119 publications with 98 being original research papers (23rd May, 2014). Figure 1 displays the research areas in which these papers have been published. There is an obvious concentration of research on $A \beta$ and Presenilin function in zebrafish.

\section{USING ZEBRAFISH TO INVESTIGATE GENES MUTATED IN FAD THE PRESENILINS}

The genes implicated in FAD have been extensively studied over the last 25 years, although our understanding of their normal functions is still far from complete. The PSEN genes 
A

B

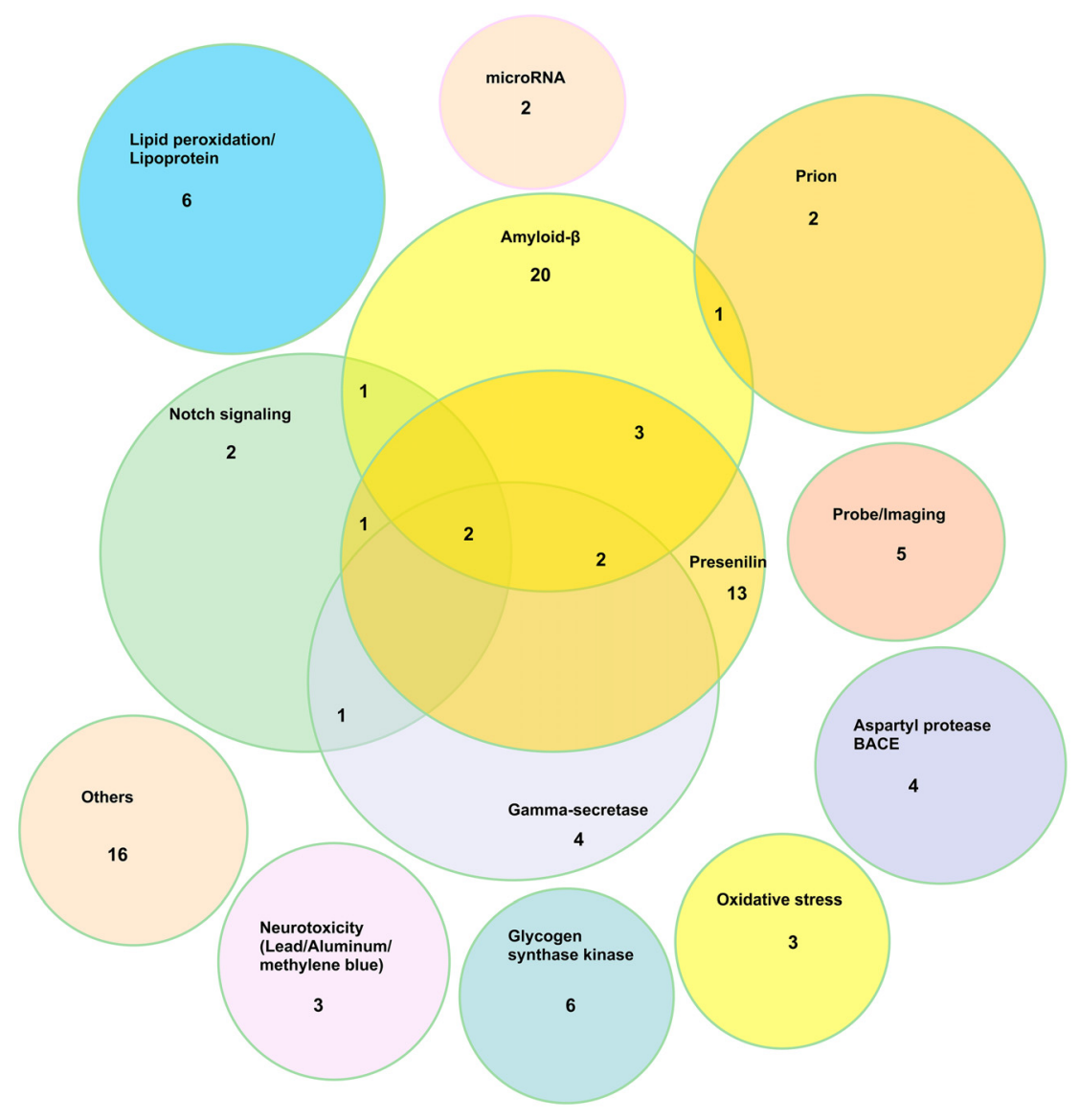

number of publications

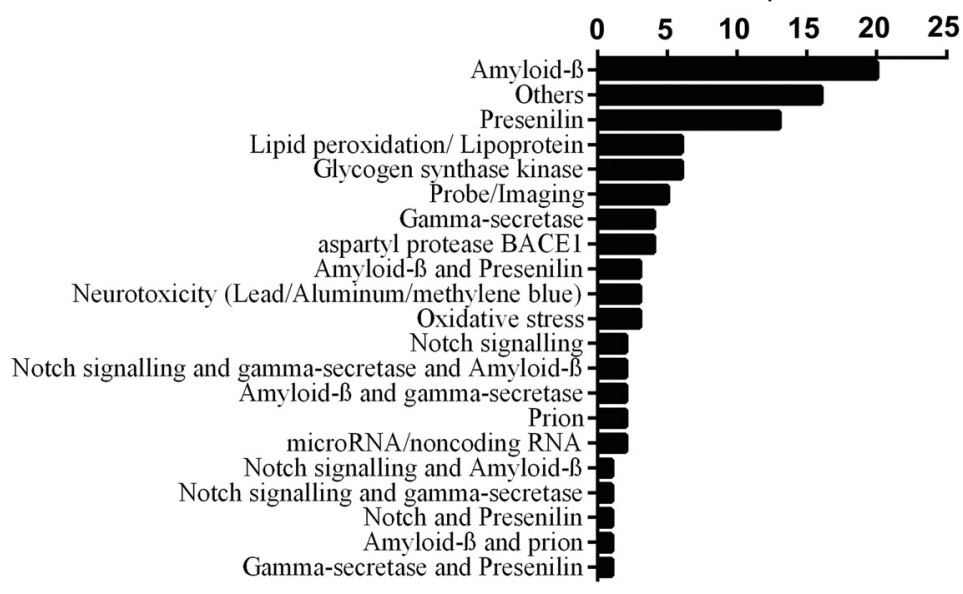

FIGURE 1 | Text mining analysis of the scientific literature to detect papers on the application of Zebrafish in Alzheimer's disease research.

The PubMed database was mined for zebrafish and Alzheimer's disease using the search term, "zebrafish+alzheimer's disease," by the MedScan Reader 5 tool implemented in the Pathway Studio 9 Package (Nikitin et al., 2003b; Novichkova etal., 2003a). MedScan Reader 5 collects information and processes undertaken data using natural language processing (NLP). The language interprets these to logical concepts and extracts functional relationships between proteins, small molecules, and cellular processes (Nikitin et al., 2003b; Novichkova et al., 2003a). 119 publications were found. Abstracts of these publications were studied and review papers were removed such that 98 papers remained. (A) Venn diagram and (B) bar graph showing numbers of research papers in different areas. A concentration of research on Amyloid $\beta$ and Presenilin function is apparent. 
play important roles in development. The zebrafish (as wellcharacterized model for studies of developmental biology) has been employed to investigate the normal functions of these genes. The $p \operatorname{sen} 1$ and $p$ sen 2 genes are ubiquitously expressed in zebrafish embryos (Leimer et al., 1999; Groth et al., 2002), implying a functional importance of these genes during development. Injection of morpholinos to knockdown presenilin gene expression revealed similar and dissimilar phenotypes compared to Presenilin knockout mice $\left(P \operatorname{sen} 1^{-/-}\right)$. Psen $1^{-/-}$mice die during development (Shen et al., 1997). To overcome this, conditional Psen 1 knockout mouse models have been developed (reviewed in van Tijn etal., 2011). Zebrafish embryos injected with a psen 1 translation-blocking morpholino are viable and have similar phenotypes (Nornes et al., 2003; Campbell et al., 2006; Nornes et al., 2008) to Psen $1^{-/-}$mice such as aberrant somite formation and Notch signaling defects (Shen et al., 1997; Wong etal., 1997) Interestingly, a zebrafish mutant (discovered in a TILLING screen) lacking Psen1 activity is viable (Sundvik et al., 2013). These mutant fish have been used to study the brains histaminergic system (Sundvik et al., 2013). This system is essential in mediating cognitive functions affected in AD. Analysis of histamine neuron numbers in the $p s e n 1^{-/-}$zebrafish brains revealed that psen 1 in zebrafish is a regulator of histaminergic neuronal development (Sundvik etal., 2013). These mutant fish will be a valuable tool for further analysis of psen 1 function in zebrafish.

Blockage of Psen2 protein translation in zebrafish embryos (Nornes et al., 2008, 2009) appears to have major effects on Notch signaling in comparison to Psen $2^{-/-}$mice which are viable and show only a minor phenotype (Herreman et al., 1999). Furthermore, loss of Psen2 expression affects the production of dorsal longitudinal ascending (DoLA) interneurons in the developing spinal cord of zebrafish larvae (Nornes et al., 2009). As loss of Psen 1 does not affect DoLAs this observation provides an in vivo assay for Psen 2 function. This assay was used to demonstrate a functional interaction between Psen1 and Psen2 in zebrafish (Nornes et al., 2009).

In the absence of technology available to analyze PSEN FAD missense mutations in zebrafish, our laboratory previously attempted to model PSEN1 splicing mutations by injecting morpholinos that target splice acceptor sites of the orthologous psen 1 gene. We attempted to cause exon skipping to exclude exon 8 or 9 from zebrafish psen 1 transcripts to mimic the effects of the human PSEN1 L271V and $\triangle 9$ mutations, respectively,. However, instead of the (at that time) expected exon exclusion, injection of morpholinos blocking splice acceptor sites lead to the failure of introns 7 and 8 to be correctly spliced out of transcripts (Nornes et al., 2008). The inclusion of intron sequence lead to premature truncation of the open reading frame (ORF) after exons 6 and 7, respectively. It appeared that the aberrant transcripts induced by morpholino injection were able to evade nonsense-mediated decay and be translated into truncated protein molecules. Assays developed in our laboratory (discussed in the next section) demonstrated that truncation of the zebrafish psen 1 transcript ORF after exons 6 and 7 had potent dominant negative effects on Psen1 activity and could also suppress Psen2 activity (Nornes et al., 2008). These putative truncated proteins were subsequently expressed in embryos by injection of synthetic mRNAs. Injection of mRNAs coding for proteins truncated after exon 6- or 7-derived sequence had the same dominant effect on Psen1 activity as the morpholino-induced aberrant psen 1 transcripts. Recently we published results where we expanded the original study to analyze a series of psen 1 ORF truncations (i.e., after exon 4, 5, 6, 7, 8; Newman et al., 2014). We found differential, dominant activation of Notch signaling and APP cleavage amongst the different truncations of Psen1. Interestingly, similar effects were also observed after injection of synthetic mRNAs encoding equivalent truncations of human PSEN1. This supports the evolutionary conservation of function of these truncated proteins. A particularly important discovery from this study was that the activity of a form of Psen1 truncated after exon 4 sequence of the ORF) cannot be observed in the absence of normal endogenous Psen1. This implies that mutant forms of PSEN1 require endogenous normal PSEN1 to exert their effects. A similar observation was made recently by Heilig et al. for various FAD mutant forms of the PSEN1 protein in mouse embryo fibroblasts (Heilig et al., 2013).

\section{OTHER COMPONENTS OF THE $\gamma$-SECRETASE COMPLEX}

There has been limited functional analysis of the zebrafish genes ortholgous to the non-PRESENILIN components of the $\gamma$-secretase complex. Blockage of translation of Psenen or Aph1b proteins in zebrafish causes phenotypes including defective somitogenesis and reduced neuron formation as expected for loss of Notch signaling (that requires $\gamma$-secretase activity; Campbell et al., 2006). Furthermore, blockage of Psenen translation leads to destabilization of Psen1 protein and causes a much greater induction of apoptosis in developing embryos than blocking translation of Psen1, Psen2, or Aph1b (Campbell et al., 2006). Simultaneous inhibition of p53 translation was able to block the induction of apoptosis (Campbell et al., 2006).

\section{THE AMYLOID-BETA PRECURSOR PROTEIN}

The zebrafish APP "co-orthologs," appa and appb have widespread and overlapping expression from mid-gastrulation in the developing embryo (Musa et al., 2001). At 24hpf both genes are expressed in the developing forebrain and other tissues with only appb expressed in the spinal cord (Musa et al., 2001). In a zebrafish study by Lee and Cole a section of $a p p b$ regulatory sequence was fused to GFP (Lee and Cole, 2007). Dissimilar to other expression studies, they observed expression of $a p p b$ in the developing vasculature. More recently, Liao et al. (2012) isolated transposon gene trap integrations that contained RFP in the appa gene and in the closely related amyloid beta precursor-like protein 2 gene (aplp2). The gene traps caused fusions to RFP of the extracellular domains of both of the encoded proteins. The fusion proteins of these genes were accumulated in the vasculature. However, they could not detect the transcripts of these genes in the endothelial cells of the vasculature. Instead, transcripts were detected in neuronal cells. This suggests that these proteins are synthesized in neuronal cells and then accumulate in the vasculature.

Translation blocking morpholinos have also been employed to investigate the function of the Appa and Appb proteins (Joshi et al., 2009). Inhibition of Appa had little effect on the developing 
embryo while Appb translation inhibition resulted in defective convergent extension cellular movements and a reduced body length. These defects could be rescued by injecting Appb deficient embryos with mRNA coding for human APP. The rescue by human APP was more effective than injection of an mRNA encoding a human APP FAD mutant (the APP Swedish double mutation $K 595 N$ and M596L). Loss of Appb activity has also been shown to cause defective neural development (Song and Pimplikar, 2012) including defective axonal out-growth patterning and synapse formation (Abramsson et al., 2013). In the study by Song and Pimplikar, only full-length human APP but not truncated forms could rescue the neuronal defects, revealing that both intracellular and extracellular domains of human APP are required for normal function (Song and Pimplikar, 2012). These studies demonstrate that zebrafish embryos can be exploited for the analysis of different mutant forms of human $A P P$.

\section{AMYLOID-BETA FUNCTION AND TOXICITY STUDIES IN ZEBRAFISH}

Although $\mathrm{A} \beta$ was discovered over 25 years ago (Goate and Hardy, 2012) and many studies have examined its claimed toxicity, the non-pathological functions of the $\mathrm{A} \beta$ peptide are still poorly understood. A study by Cameron et al. (2012) demonstrated that high levels of $A \beta$ can increase cerebrovascular branching in the developing zebrafish hindbrain. They then completed a follow up study to determine whether lowering $A \beta$ levels would have the opposite effect (Luna et al., 2013). From 2 days post fertilization (dpf) APP-deficient (by morpholino injection) and $A \beta$ deficient (endogenous $A \beta$ production blocked using a $\beta$-secretase inhibitor) larvae presented with cerebrovascular defects. Interestingly, these defects could be rescued by treating the embryos with the human $A \beta-42$ peptide but not the shorter cleavage product of APP named p3 (that arises from cleavage of APP by $\alpha$-secretase and $\gamma$-secretase). These results suggest that $A \beta$ may play a role in maintaining normal cerebrovascular function. This interesting finding using the zebrafish model provided further evidence that caution should be taken when treating $\mathrm{AD}$ with drugs designed to inhibit $A \beta$ production such as BSIs and $\gamma$-secretase inhibitors (GSIs).

As mentioned above the amyloid hypothesis of $\mathrm{AD}$ proposes that aggregations of $\mathrm{A} \beta$ peptides are toxic. Therefore, there has been a considerable effort put towards developing and discovering $A \beta$-lowering compounds to counter the toxicity. Inhibition of $\beta$-secretase, also known as beta-site APP cleaving enzyme (BACE1), is of prime interest for the development of amyloidlowering drugs. BACE1 has become an attractive target for drug development as, in comparison to the lethality observed in Psen 1 null mice, Bace1 knock-out mice are viable (Roberds et al., 2001; Luo etal., 2003) and have subtle phenotypes (Harrison et al., 2003; Willem et al., 2006). Furthermore no phenotypes have been reported for knockout of the Bace1 homolog, Bace 2 and deletion of Bace 2 in mice did alter $A \beta$ generation (Dominguez et al., 2005). Recently, bace1 and bace2 loss-of-function mutations have been introduced into the zebrafish genome by genome editing using ZFNs (van Bebber etal., 2013). bace1 mutants had an increased number of mechanosensory neuromasts and, similar to observations in the mouse, Bacel knockout caused a decrease in myelination. bace 2 mutants had a distinct melanocyte migration phenotype that was not observed in the bace1 mutants. There was no effect on myelination or neuromasts in the bace2 mutants. The phenotypes observed in the single mutants were not enhanced further in the Bace1/2 double knockout. Together the data from this study suggests that, in zebrafish, Bace1 and Bace2, have distinct non-redundant physiological functions. These specific phenotypes observed in zebrafish bace1 and bace 2 mutants have provided important information on BACE1 and BACE2 function and support that zebrafish can be a useful in vivo system for determining, e.g., whether BACE1 inhibitors also inhibit BACE2 function and vice versa. In vivo evaluation of BACE inhibitors is integral for establishing these drugs as a therapeutic option for $\mathrm{AD}$.

The zebrafish is an excellent system for the screening of chemical libraries. Embryos and larvae placed into microtiter plates can be treated with various chemicals in their aqueous support medium. This strategy can be employed to reveal therapeutic compounds for various disease states modeled in the fish. The only published study that has generated a transgenic $\mathrm{A} \beta$ toxicity model in zebrafish involved fusing the human $\mathrm{A} \beta-42$ sequence to the promoter of the mitfa (nacre) gene (Newman et al., 2010). This would drive expression of human $A \beta-42$ specifically in the melanincontaining pigment cells (melanocytes) of the zebrafish in the hope that it would produce an easily identifiable disrupted pigmentation pattern phenotype without being lethal to the zebrafish larvae. A disrupted pattern did become evident in the adult fish. However, unfortunately it only became apparent after 16 months which is too late for drug screening and was too late for breeding the old, infertile fish. $A \beta$ toxicity can also be analyzed in zebrafish simply by exposing embryos to amyloid-beta in their supportive aqueous environment. Treatment of embryos with $2.5 \mu \mathrm{M}$ $\mathrm{A} \beta$-40 caused defective development including that of the vasculature and also accelerated cell senescence (Donnini et al., 2010). This system could be expanded to test $A \beta-42$ (more aggregative form) and also to find compounds that ameliorate these observed defects.

$\gamma$-secretase inhibitors (GSIs) have been investigated as a therapeutic option to inhibit the production of $A \beta$ peptides. However, the use of GSIs comes with the potential side affects of also affecting Notch signaling. Testing the toxicity of GSIs on zebrafish has revealed important information on how Notch related pathways are adversely affected by GSIs (Yang et al., 2010).

\section{ASSAYS FOR AD-RELEVANT CELLULAR PATHWAYS AND PROCESSES IN ZEBRAFISH $\gamma$-SECRETASE ACTIVITY ASSAYS}

There are a number of cellular pathways and processes that are aberrant in AD. The zebrafish are a useful system for investigating molecular events such as $\gamma$-secreatse activity and autophagy that have been implicated in $\mathrm{AD}$ pathogenesis.

There are over 70 proteins that are known to be substrates of $\gamma$-secreatse activity (Lleo and Saura, 2011). In zebrafish, $\gamma$-secretase activity was initially analyzed by observing changes in the expression of genes known to be downstream targets of Notch signaling such as hairy-related 6 (Bernardos et al., 2005; Campbell et al., 2006; Arslanova et al., 2010) and neurogenin (Campbell et al., 
2006). However, gene expression can be under different transcriptional control in different regions of the embryo (Campbell et al., 2006; Arslanova et al., 2010). Therefore, using whole embryos in quantitative PCR analysis is not informative to assess changes in gene expression in response to various factors. To overcome this, changes in gene expression in a particular region of the embryos have been assessed by whole-mount in situ transcript hybridization (Campbell et al., 2006; Nornes et al., 2008; Arslanova et al., 2010). However, this is not normally used as a quantitative technique since many variables can influence the strength of the staining signal such as fixation conditions and incubation times. Consequently, use of this technique to assay relative differences in Notch signals under various treatments requires stringent controls.

Recently, the first assay to assess directly $\gamma$-secretase cleavage activity was developed by Wilson and Lardelli (2013). $\alpha$ - and $\beta$-secretase cleavage of Appa provide substrates for subsequent $\gamma$-secretase cleavage. Unfortunately these cleaved forms of Appa cannot be detected in zebrafish embryos prior to $48 \mathrm{hpf}$ which currently makes monitoring endogenous Appa cleavage in manipulated zebrafish embryos difficult. Therefore, a fragment of Appa equivalent to the membrane-embedded remnant of APP following $\beta$-secretase cleavage was fused to GFP and expressed transiently in zebrafish embryos by the use of Tol2 vector transgenesis system. This construct is co-expressed with a set ratio of free GFP (for signal normalization). Western immunoblotting is then used to assess the ratio of the Appa:GFP substrate to free GFP (the $\gamma$-secretase cleavage product itself is too unstable to be observed). Once an Appa:GFP/ free GFP ratio is determined for a protein sample from a pool of manipulated embryos (e.g., drug treatment, morpholino or mRNA injection) it can then be compared to control embryos to determine how that particular manipulation is affecting $\gamma$-secretase cleavage activity.

\section{ASSAYING PROTEIN DEGRADATION PATHWAYS IN ZEBRAFISH}

Excess or aberrant cellular proteins can be degraded by the ubiquitin-proteasome system (UPS). Since protein aggregation is implicated in many neurodegenerative diseases (Ross and Poirier, 2004) it is unsurprising that problems with UPS function have been implicated in neurodegenerative disease such as AD (West et al., 1994; Nikitin etal., 2003a; Novichkova et al., 2003b) and Parkinson's disease (Olanow and McNaught, 2006). Zebrafish have been used to investigate UPS function in Parkinson's disease (Regeur et al., 1994); however, no analyses in zebrafish have yet examined the UPS with respect to AD.

Autophagy is an important mechanism required for the degradation of dysfunctional and unwanted cellular components (including incorrectly folded and aggregated proteins) through the actions of lysosomes. Indeed, autophagy has been identified as a pathway for the degradation of accumulated $A \beta$ peptides (Nilsson et al., 2013). Recently, the Presenilin proteins were suggested to have a major role in autophagy with FAD mutations in human PSEN1 inhibiting this function (Lee et al., 2010). These authors presented evidence showing that PSEN1 acts as a chaperone in the ER for a transmembrane protein required for acidification of the lysosomes, the v-ATPase V0al subunit [Lee etal., 2010; however, other reports have disputed this finding (Coen et al.,
2012; Zhang etal., 2012)]. They also demonstrated that this function of PSEN1 is dependent on the full-length PSEN1 holoprotein rather than the endoproteolysed form that is active in the $\gamma$-secretase complex. Furthermore, a $\gamma$-secretase inhibitor and loss of another $\gamma$-secretase complex component (NCT) had no effect on autophagy (Lee et al., 2010) suggesting that this function of PSEN1 is independent from its function in $\gamma$-secretase complexes.

Autophagy can be analyzed in zebrafish by observing induction of the LC3-II protein by western immunoblotting using a human antibody against LC3 that cross-reacts with zebrafish Lc3 (He et al., 2009; He and Klionsky, 2010). Transgenic zebrafish have also been developed that express GFP fused to Lc3 (He et al., 2009). As Presenilin protein expression can easily be manipulated in the zebrafish these autophagy assay are a useful tool for further investigation of the involvement of the Presenilins in autophagy.

\section{USING THE ZEBRAFISH TO INVESTIGATE OTHER ASPECTS OF ALZHEIMER'S DISEASE ETIOLOGY ANALYSIS OF HYPOXIA IN THE ZEBRAFISH}

There is accumulating evidence suggesting that hypoxia is an important initiating factor in the pathogenesis of AD. Under hypoxic conditions the electron transport chain in the mitochondria increases free radical production that leads to increased oxidative stress (Bell et al., 2007). Biomarkers of hypoxia can differentiate between people with mild cognitive impairment that progress to $\mathrm{AD}$ and those who do not (Oresic et al., 2011). The risk factors for cardiovascular disease and $\mathrm{AD}$ are similar (Kotze and van Rensburg, 2012) and it is anticipated that vasculature problems would affect oxygenation of the brain. Interestingly, $A \beta$ levels in serum have been shown to be elevated after cardiac arrest (Zetterberg et al., 2011).

Zebrafish are an advantageous system for analysis of the effects of hypoxia on various biological functions. Zebrafish embryos and adults can be exposed to real hypoxia by depleting their water environment of oxygen or to chemical mimicry of hypoxia through, e.g., sodium azide treatment (Moussavi Nik et al., 2011). Similarly to what is observed in humans (Lukiw et al., 2001; Pluta, 2005; De Gasperi et al., 2010; Zhang and Le, 2010), hypoxia upregulates psen1, psen2, $a p p a, a p p b$, and bacel in zebrafish adult brain and larvae (Moussavi Nik etal., 2012). This suggests that $A \beta$ is produced as a protective response to hypoxia in both human and zebrafish cells - a response conserved over 450 Mya of evolutionary time. Note, however, that while all the enzymes required to cleave $A \beta$ from Appa and/or Appb are present in zebrafish, the existence of $A \beta$ itself has not yet been directly demonstrated (e.g., through immunoblotting or mass spectrometry). The study by Moussavi Nik et al. (2012) also demonstrated that, unlike in mammals, F2-isoprostanes are not a good marker of oxidative stress in zebrafish and that the upregulation of catalase gene expression can be a better alternative marker for demonstration of oxidative stress in zebrafish (Jin et al., 2011; Tseng et al., 2013).

\section{APOE}

The APOE $\varepsilon 4$ allele has been identified has the main genetic risk factor for SAD. APOE is important for clearance of amyloid-beta 
from the brain (Huang and Mucke, 2012), while the AD riskassociated $\varepsilon 4$ allele has been shown to impair the clearance of $\mathrm{A} \beta$ (Deane et al., 2008) and, more recently, to affect the integrity of the blood-brain barrier (Bell et al., 2012). There has been little research investigating APOE function in zebrafish. Expression studies of apoea (Raymond et al., 2006) and apoeb (Pujic et al., 2006) revealed expression in the developing retina and yolk syncytial layer. Furthermore, apoeb has also been observed in microglial cells (Veth et al., 2011), developing fins and epidermis (Monnot et al., 1999; Tingaud-Sequeira et al., 2006), regenerating fin tissue (Monnot et al., 1999), macrophages (Lien et al., 2006), liver, intestine, and ovary (Levi et al., 2012).

\section{MAPT}

The MAPT is the main component of the NFTs found in AD brains. Various dysfunctions of the tau protein are found in other neurodegenerative disorders such as frontotemporal dementia (FTD), corticobasal degeneration and progressive supranuclear palsy (Pittman et al., 2006). Diseases with tau-like pathology are collectively termed "tauopathies." A review by Bai and Burton (2011) discussed how the zebrafish has been used to investigate these diseases. A number of MAPT protein isoforms exist as a result of alternative splicing of MAPT transcripts. These isoforms can be classified into two groups, $3 \mathrm{R}$ or $4 \mathrm{R}$, depending on the number of tubulin-binding motifs. It appears that an overall one-to-one ratio of $3 \mathrm{R}$ to $4 \mathrm{R}$ transcripts is required for normal functioning of the MAPT protein in the brain (Goedert and Spillantini, 2006). In most tauopathies this ratio is found to be changed (Goedert and Spillantini, 2006) and altered splicing of MAPT is also suggested to occur in AD brains (Conrad et al., 2007).

Transgenic zebrafish expressing human MAPT were generated and investigated prior to identification of the zebrafish ortholog(s) of MAPT. In these studies human MAPT was specifically expressed in zebrafish CNS neurons (Bai et al., 2007; Paquet et al., 2009). Bai et al. used the promoter of the enolase 2 gene to drive the expression of MAPT $4 \mathrm{R}$ in zebrafish neurons at approximately eightfold higher levels than what is observed in human brain. This resulted in accumulations of tau protein (resembling NFTs) in the zebrafish brain. In the study by Paquet et al., the $H u C$ promoter was employed to drive expression of a Gal4:VP16 fusion protein in neurons. This protein was then bound to UAS sites in a bidirectional promoter transcribing the DsRed fluorescent marker protein gene and a mutant form of human MAPT associated with FTD, TAU-P301L. The transgenic zebrafish larvae showed biochemical changes consistent with those observed in human tauopathies. However, it should be noted that no comparisons of phenotype were made between the nonmutant and mutant forms of human MAPT in the zebrafish. Furthermore, the expression levels of the transgenes relative to the endogenous zebrafish mapt genes were not assessed in these studies. Despite these limitations these transgenic zebrafish models provide a useful system to investigate whether chemical inhibitors can modulate the observed tauopathy-associated changes.

The zebrafish "co-orthologs" of the human MAPT gene, mapta and maptb have similar but not completely overlapping patterns of expression in developing embryos (Chen et al., 2009). They are both predominantly expressed in the developing CNS while only $m a p t b$ has strong expression in the trigeminal ganglion and dorsal sensory neurons of the spinal cord. Mapta is spliced into 4R$6 \mathrm{R}$ isoforms while maptb is spliced mainly into $3 \mathrm{R}$ isoforms. This expression is in contrast to mouse Mapt which is mainly expressed as $3 \mathrm{R}$ in the brain (and hence may not be a good model of MAPT function for human pathologies). Manipulation of the expression of the zebrafish mapt isoforms may therefore be advantageous for understanding the function of $3 \mathrm{R}$ and $4 \mathrm{R}$ MAPT and the role(s) the $3 \mathrm{R}: 4 \mathrm{R}$ ratio plays in pathogenesis.

\section{THE FUTURE FOR MODELING ALZHEIMER'S DISEASE IN ZEBRAFISH}

The zebrafish is rapidly emerging as an attractive model for AD research. They are an ideal model for drug testing prior to clinical testing in rodents. However, there are still aspects of this model that require better understanding. For the zebrafish system to be used to model aspects of AD pathobiology we need to better understand zebrafish brain structure and function and also gain a deeper understanding of adult zebrafish brain physiology. Work so far has revealed that the zebrafish brain does have a reasonable level of conservation of basic structure when compared to mammals as well as similar neuroanatomical and neurochemical pathways to those that play roles in human disease (reviewed in Santana et al., 2012). We have revealed various aspects of presenilin gene biology using the zebrafish that would otherwise be difficult to observe/analyze in other models. However, to analyze effectively future transgenic and mutant zebrafish models of $\mathrm{AD}$ we need to strengthen our understanding of the functions in zebrafish of some of the orthologs of the key genes implicated in human AD pathogenesis such as MAPT and APOE.

Whether the zebrafish can be employed to model a late-onset disease like $\mathrm{AD}$ is debatable since zebrafish have a profound capacity for regeneration and this must impact on the development of neurodegenerative phenotypes. Neurogenesis in the adult zebrafish brain is much more abundant than is observed in mammals (Kizil et al., 2012) consequently making analysis of neuronal loss difficult. Despite these limitations the recent availability and feasibility of using genome editing technologies presents an exciting opportunity to develop zebrafish genetic models of neurodegenerative diseases such as AD. ZFNs, TALENs and CRISP/Rs have been validated for use in the zebrafish [reviewed by Hwang et al., 2013; Schmid and Haass, 2013) and it is inevitable that FAD mutations will be introduced into zebrafish FAD gene orthologs.

Animal models are a useful tool in investigating the causes and pathologies of human diseases. Obviously such models can never reflect the complete pathology that is observed in human cases. The complexity of the human brain makes AD a particularly difficult disease to model in animals. However, by using a number of different models including the zebrafish, we can exploit the unique characteristics of each to unravel the molecular basis of this disease.

\section{REFERENCES}

Abramsson, A., Kettunen, P., Banote, R. K., Lott, E., Li, M., Arner, A., et al. (2013). The zebrafish amyloid precursor protein-b is required for motor neuron guidance and synapse formation. Dev. Biol. 381, 377-388. doi: 10.1016/j.ydbio.2013. 06.026 
Arslanova, D., Yang, T., Xu, X. Y., Wong, S. T., Augelli-Szafran, C. E., and Xia, W. M. (2010). Phenotypic analysis of images of zebrafish treated with Alzheimer's gamma-secretase inhibitors. BMC Biotechnol. 10:24. doi: 10.1186/1472-6750$10-24$

Babin, P. J., Thisse, C., Durliat, M., Andre, M., Akimenko, M. A., and Thisse, B. (1997). Both apolipoprotein E and A-I genes are present in a nonmammalian vertebrate and are highly expressed during embryonic development. Proc. Natl. Acad. Sci. U.S.A. 94, 8622-8627. doi: 10.1073/pnas.94. 16.8622

Bai, Q., and Burton, E. A. (2011). Zebrafish models of tauopathy. Biochim. Biophys. Acta 1812, 353-363. doi: 10.1016/j.bbadis.2010.09.004

Bai, Q., Garver, J. A., Hukriede, N. A., and Burton, E. A. (2007). Generation of a transgenic zebrafish model of Tauopathy using a novel promoter element derived from the zebrafish eno2 gene. Nucleic Acids Res. 35, 6501-6516. doi 10.1093/nar/gkm608

Bell, E. L., Klimova, T. A., Eisenbart, J., Moraes, C. T., Murphy, M. P., Budinger, G. R. S., et al. (2007). The Q(o) site of the mitochondrial complex III is required for the transduction of hypoxic signaling via reactive oxygen species production. J. Cell Biol. 177, 1029-1036. doi: 10.1083/jcb.200609074

Bell, R. D., Winkler, E. A., Singh, I., Sagare, A. P., Deane, R., Wu, Z., et al. (2012). Apolipoprotein E controls cerebrovascular integrity via cyclophilin A. Nature 485, 512-516. doi: 10.1038/nature11087

Berger, J., Berger, S., Jacoby, A. S., Wilton, S. D., and Currie, P. D. (2011). Evaluation of exon-skipping strategies for Duchenne muscular dystrophy utilizing dystrophin-deficient zebrafish. J. Cell Mol. Med. 15, 2643-2651. doi: 10.1111/j.1582-4934.2011.01260.x

Bernardos, R. L., Lentz, S. I., Wolfe, M. S., and Raymond, P. A. (2005). Notch-Delta signaling is required for spatial patterning and Muller glia differentiation in the zebrafish retina. Dev. Biol. 278, 381-395. doi: 10.1016/j.ydbio.2004.11.018

Blennow, K., De Leon, M. J., and Zetterberg, H. (2006). Alzheimer's disease. Lancet 368, 387-403. doi: 10.1016/S0140-6736(06)69113-7

Cameron, D. J., Galvin, C., Alkam, T., Sidhu, H., Ellison, J., Luna, S., et al. (2012). Alzheimer's-related peptide amyloid-beta plays a conserved role in angiogenesis. PLOS ONE 7:e39598. doi: 10.1371/journal.pone.0039598

Campbell, W. A., Yang, H., Zetterberg, H., Baulac, S., Sears, J. A., Liu, T., et al. (2006). Zebrafish lacking Alzheimer presenilin enhancer 2 (Pen-2) demonstrate excessive p53-dependent apoptosis and neuronal loss. J. Neurochem. 96, 1423-1440. doi: 10.1111/j.1471-4159.2006.03648.x

Catchen, J. M., Braasch, I., and Postlethwait, J. H. (2011). Conserved synteny and the zebrafish genome. Methods Cell Biol. 104, 259-285. doi: 10.1016/B978-0-12374814-0.00015-X

Chen, M., Martins, R. N., and Lardelli, M. (2009). Complex splicing and neural expression of duplicated tau genes in zebrafish embryos. J. Alzheimers Dis. 18, 305-317. doi: 10.3233/JAD-2009-1145

Coen, K., Flannagan, R. S., Baron, S., Carraro-Lacroix, L. R., Wang, D., Vermeire, W., et al. (2012). Lysosomal calcium homeostasis defects, not proton pump defects, cause endo-lysosomal dysfunction in PSEN-deficient cells. J. Cell Biol. 198, 23-35. doi: $10.1083 /$ jcb.201201076

Conrad, C., Zhu, J., Conrad, C., Schoenfeld, D., Fang, Z., Ingelsson, M., et al. (2007). Single molecule profiling of tau gene expression in Alzheimer's disease. J. Neurochem. 103, 1228-1236. doi: 10.1111/j.1471-4159.2007.04857.x

De Gasperi, R., Sosa, M. A., Dracheva, S., and Elder, G. A. (2010). Presenilin-1 regulates induction of hypoxia inducible factor-lalpha: altered activation by a mutation associated with familial Alzheimer's disease. Mol. Neurodegener. 5:38. doi: 10.1186/1750-1326-5-38

Deane, R., Sagare, A., Hamm, K., Parisi, M., Lane, S., Finn, M. B., et al. (2008). apoE isoform-specific disruption of amyloid beta peptide clearance from mouse brain. J. Clin. Invest. 118, 4002-4013. doi: 10.1172/JCI36663 36663

Dominguez, D., Tournoy, J., Hartmann, D., Huth, T., Cryns, K., Deforce, S., et al. (2005). Phenotypic and biochemical analyses of BACE1- and BACE2-deficient mice. J. Biol. Chem. 280, 30797-30806. doi: 10.1074/jbc.M505249200

Donnini, S., Solito, R., Cetti, E., Corti, F., Giachetti, A., Carra, S., et al. (2010). A beta peptides accelerate the senescence of endothelial cells in vitro and in vivo, impairing angiogenesis. FASEB J. 24, 2385-2395. doi: 10.1096/Fj.09146456

Draper, B. W., Morcos, P. A., and Kimmel, C. B. (2001). Inhibition of zebrafish fgf8 pre-mRNA splicing with morpholino oligos: a quantifiable method for gene knockdown. Genesis 30, 154-156. doi: 10.1002/gene.1053
Francis, R., Mcgrath, G., Zhang, J. H., Ruddy, D. A., Sym, M., Apfeld, J., et al. (2002). aph-1 and pen-2 are required for notch pathway signaling, gamma-secretase cleavage of beta APP, and presenilin protein accumulation. Dev. Cell 3, 85-97. doi: 10.1016/S1534-5807(02)00189-2

Goate, A., and Hardy, J. (2012). Twenty years of Alzheimer's disease-causing mutations. J. Neurochem. 120(Suppl. 1), 3-8. doi: 10.1111/j.1471-4159.2011.07575.x

Goedert, M., and Spillantini, M. G. (2006). A century of Alzheimer's disease. Science 314, 777-781. doi: 10.1126/science.1132814

Groth, C., Nornes, S., Mccarty, R., Tamme, R., and Lardelli, M. (2002). Identification of a second presenilin gene in zebrafish with similarity to the human Alzheimer's disease gene presenilin2. Dev. Genes Evol. 212, 486-490. doi: 10.1007/s00427002-0269-5

Halpern, M. E., Rhee, J., Goll, M. G., Akitake, C. M., Parsons, M., and Leach, S. D. (2008). Gal4/UAS transgenic tools and their application to zebrafish. Zebrafish 5, 97-110. doi: 10.1089/zeb.2008.0530

Hans, S., Kaslin, J., Freudenreich, D., and Brand, M. (2009). Temporallycontrolled site-specific recombination in zebrafish. PLoS ONE 4:e4640. doi: 10.1371/journal.pone.0004640

Hardy, J. A., and Higgins, G. A. (1992). Alzheimer's disease: the amyloid cascade hypothesis. Science 256, 184-185 doi: 10.1126/science.1566067

Harrison, S. M., Harper, A. J., Hawkins, J., Duddy, G., Grau, E., Pugh, P. L., et al. (2003). BACE1 (beta-secretase) transgenic and knockout mice: identification of neurochemical deficits and behavioral changes. Mol. Cell. Neurosci. 24, 646-655. doi: 10.1016/S1044-7431(03)00227-6

He, C., Bartholomew, C. R., Zhou, W., and Klionsky, D. J. (2009). Assaying autophagic activity in transgenic GFP-Lc3 and GFP-Gabarap zebrafish embryos. Autophagy 5, 520-526. doi: 10.4161/auto.5.4.7768

He, C., and Klionsky, D. J. (2010). Analyzing autophagy in zebrafish. Autophagy 6, 642-644. doi: 10.4161/auto.6.5.12092

Heilig, E. A., Gutti, U., Tai, T., Shen, J., and Kelleher, R. J. (2013). Trans-dominant negative effects of pathogenic PSEN1 mutations on gamma-secretase activity and Abeta production. J. Neurosci. 33, 11606-11617. doi: 10.1523/JNEUROSCI.095413.2013

Herreman, A., Hartmann, D., Annaert, W., Saftig, P., Craessaerts, K., Serneels, L., et al. (1999). Presenilin 2 deficiency causes a mild pulmonary phenotype and no changes in amyloid precursor protein processing but enhances the embryonic lethal phenotype of presenilin 1 deficiency. Proc. Natl. Acad. Sci. U.S.A. 96, 11872-11877. doi: 10.1073/pnas.96.21.11872

Hisano, Y., Ota, S., and Kawahara, A. (2014). Genome editing using artificial site-specific nucleases in zebrafish. Dev. Growth Differ. 56, 26-33. doi: $10.1111 /$ dgd.12094

Huang, Y., and Mucke, L. (2012). Alzheimer mechanisms and therapeutic strategies. Cell 148, 1204-1222. doi: 10.1016/j.cell.2012.02.040

Hwang, W. Y., Fu, Y., Reyon, D., Maeder, M. L., Tsai, S. Q., Sander, J. D., et al. (2013). Efficient genome editing in zebrafish using a CRISPR-Cas system. Nat. Biotechnol. 31, 227-229. doi: 10.1038/nbt.2501

Jin, Y. X., Zheng, S. S., Pu, Y., Shu, L. J., Sun, L. W., Liu, W. P., et al. (2011). Cypermethrin has the potential to induce hepatic oxidative stress, DNA damage and apoptosis in adult zebrafish (Danio rerio). Chemosphere 82, 398-404. doi: 10.1016/j.chemosphere.2010.09.072

Joshi, P., Liang, J. O., Dimonte, K., Sullivan, J., and Pimplikar, S. W. (2009). Amyloid precursor protein is required for convergent-extension movements during zebrafish development. Dev. Biol. 335, 1-11. doi: 10.1016/j.ydbio.2009. 07.041

Kawakami, K., Shima, A., and Kawakami, N. (2000). Identification of a functional transposase of the Tol2 element, an Ac-like element from the Japanese medaka fish, and its transposition in the zebrafish germ lineage. Proc. Natl. Acad. Sci. U.S.A. 97, 11403-11408. doi: 10.1073/pnas.97.21.11403

Kizil, C., Kaslin, J., Kroehne, V., and Brand, M. (2012). Adult neurogenesis and brain regeneration in zebrafish. Dev. Neurobiol. 72, 429-461. doi: 10.1002/dneu.20918

Kotze, M. J., and van Rensburg, S. J. (2012). Pathology supported genetic testing and treatment of cardiovascular disease in middle age for prevention of Alzheimer's disease. Metab. Brain Dis. 27, 255-266. doi: 10.1007/s11011-012-9296-9298

Kumar, S., and Hedges, S. B. (1998). A molecular timescale for vertebrate evolution. Nature 392, 917-920. doi: 10.1038/31927

Lee, J. A., and Cole, G. J. (2007). Generation of transgenic zebrafish expressing green fluorescent protein under control of zebrafish amyloid precursor protein gene regulatory elements. Zebrafish 4, 277-286. doi: 10.1089/zeb.2007.0516 
Lee, J. H., Yu, W. H., Kumar, A., Lee, S., Mohan, P. S., Peterhoff, C. M., et al. (2010). Lysosomal proteolysis and autophagy require presenilin 1 and are disrupted by Alzheimer-related PS1 mutations. Cell 141, 1146-1158. doi: 10.1016/j.cell.2010.05.008

Leimer, U., Lun, K., Romig, H., Walter, J., Grunberg, J., Brand, M., et al. (1999). Zebrafish (Danio rerio) presenilin promotes aberrant amyloid betapeptide production and requires a critical aspartate residue for its function in amyloidogenesis. Biochemistry 38, 13602-13609. doi: 10.1021/bi99 $1453 n$

Levi, L., Ziv, T., Admon, A., Levavi-Sivan, B., and Lubzens, E. (2012). Insight into molecular pathways of retinal metabolism, associated with vitellogenesis in zebrafish. Am. J. Physiol. Endocrinol. Metab. 302, E626-E644. doi: 10.1152/ajpendo.00310.2011

Liao, H. K., Wang, Y., Watt, K. E. N., Wen, Q., Breitbach, J., Kemmet, C. K., et al (2012). Tol2 gene trap integrations in the zebrafish amyloid precursor protein genes appa and aplp2 reveal accumulation of secreted APP at the embryonic veins. Dev. Dyn. 241, 415-425. doi: 10.1002/Dvdy.23725

Lien, C. L., Schebesta, M., Makino, S., Weber, G. J., and Keating, M. T. (2006). Gene expression analysis of zebrafish heart regeneration. PLoS Biol. 4:e260. doi 10.1371/journal.pbio.0040260

Lieschke, G. J., and Currie, P. D. (2007). Animal models of human disease: zebrafish swim into view. Nat. Rev. Genet. 8, 353-367. doi: 10.1038/nrg2091

Lleo, A., and Saura, C. A. (2011). Gamma-secretase substrates and their implications for drug development in Alzheimer's disease. Curr. Top. Med. Chem. 11, 15131527. doi: $10.2174 / 156802611795861004$

Lukiw, W. J., Gordon, W. C., Rogaev, E. I., Thompson, H., and Bazan N. G. (2001). Presenilin-2 (PS2) expression up-regulation in a model of retinopathy of prematurity and pathoangiogenesis. Neuroreport 12, 53-57 doi: 10.1097/00001756-200101220-00019

Luna, S., Cameron, D. J., and Ethell, D. W. (2013). Amyloid-beta and APP deficiencies cause severe cerebrovascular defects: important work for an old villain. PLoS ONE 8:e75052. doi: 10.1371/journal.pone.0075052

Luo, Y., Bolon, B., Damore, M. A., Fitzpatrick, D., Liu, H., Zhang, J., et al. (2003). BACE1 (beta-secretase) knockout mice do not acquire compensatory gene expression changes or develop neural lesions over time. Neurobiol. Dis. 14, 81-88. doi: 10.1016/S0969-9961(03)00104-9

Marchesi, V. T. (2011). Alzheimer's dementia begins as a disease of small blood vessels, damaged by oxidative-induced inflammation and dysregulated amyloid metabolism: implications for early detection and therapy. FASEB J. 25, 5-13. doi: 10.1096/fj.11-0102ufm

Martins, I. J., Hone, E., Foster, J. K., Sunram-Lea, S. I., Gnjec, A., Fuller, S. J., et al. (2006). Apolipoprotein E, cholesterol metabolism, diabetes, and the convergence of risk factors for Alzheimer's disease and cardiovascular disease. Mol. Psychiatry 11, 721-736. doi: 10.1038/sj.mp.4001854

McCarthy, J. V., Twomey, C., and Wujek, P. (2009). Presenilin-dependent regulated intramembrane proteolysis and gamma-secretase activity. Cell Mol. Life. Sci. 66 1534-1555. doi: 10.1007/s00018-009-8435-9

Monnot, M. J., Babin, P. J., Poleo, G., Andre, M., Laforest, L., Ballagny, C., etal. (1999). Epidermal expression of apolipoprotein E gene during fin and scale development and fin regeneration in zebrafish. Dev. Dyn. 214, 207-215. doi: 10.1002/(SICI)1097-0177(199903)214:3<207::AID-AJA4>3.0. $\mathrm{CO} ; 2-5$

Moussavi Nik, S. H., Newman, M., and Lardelli, M. (2011). The response of HMGA1 to changes in oxygen availability is evolutionarily conserved. Exp. Cell Res. 317, 1503-1512. doi: 10.1016/j.yexcr.2011.04.004

Moussavi Nik, S. H., Wilson, L., Newman, M., Croft, K., Mori, T. A., Musgrave, I., et al. (2012). The BACE1-PSEN-AbetaPP regulatory axis has an ancient role in response to low oxygen/oxidative stress. J. Alzheimers Dis. 28, 515-530. doi: 10.3233/JAD-2011-110533

Musa, A., Lehrach, H., and Russo, V. A. (2001). Distinct expression patterns of two zebrafish homologues of the human APP gene during embryonic development. Dev. Genes Evol. 211, 563-567. doi: 10.1007/s00427-001-0189-9

Newman, M., Nornes, S., Martins, R. N., and Lardelli, M. T. (2012). Robust homeostasis of Presenilin1 protein levels by transcript regulation. Neurosci. Lett. 519 14-19. doi: 10.1016/j.neulet.2012.04.064

Newman, M., Wilson, L., Camp, E., Verdile, G., Martins, R., and Lardelli, M. (2010). A zebrafish melanophore model of amyloid beta toxicity. Zebrafish 7, 155-159. doi: $10.1089 /$ zeb. 2009.0628
Newman, M., Wilson, L., Verdile, G., Lim, A., Khan, I., Moussavi Nik, S. H., et al (2014). Differential, dominant activation and inhibition of Notch signalling and APP cleavage by truncations of PSEN1 in human disease. Hum. Mol. Genet. 23, 602-617. doi: 10.1093/hmg/ddt448

Nikitin, A., Egorov, S., Daraselia, N., and Mazo, I. (2003a). Pathway studio-the analysis and navigation of molecular networks. Bioinformatics 19, 2155-2157. doi: 10.1093/bioinformatics/btg290

Nikitin, A., Egorov, S., Daraselia, N., and Mazo, I. (2003b). Pathway studio-the analysis and navigation of molecular networks. Bioinformatics 19, 2155-2157. doi: 10.1093/bioinformatics/btg290

Nilsson, P., Loganathan, K., Sekiguchi, M., Matsuba, Y., Hui, K., Tsubuki, S., et al. (2013). Abeta secretion and plaque formation depend on autophagy. Cell Rep. 5, 61-69. doi: 10.1016/j.celrep.2013.08.042

Nornes, S., Groth, C., Camp, E., Ey, P., and Lardelli, M. (2003). Developmental control of Presenilin 1 expression, endoproteolysis, and interaction in zebrafish embryos. Exp. Cell Res. 289, 124-132. doi: 10.1016/S0014-4827(03) 00257-X

Nornes, S., Newman, M., Verdile, G., Wells, S., Stoick-Cooper, C. L., Tucker, B., et al. (2008). Interference with splicing of Presenilin transcripts has potent dominant negative effects on Presenilin activity. Hum. Mol. Genet. 17, 402-412. doi: $10.1093 / \mathrm{hmg} / \mathrm{ddm} 317$

Nornes, S., Newman, M., Wells, S., Verdile, G., Martins, R. N., and Lardelli, M. (2009). Independent and cooperative action of Psen 2 with Psen 1 in zebrafish embryos. Exp. Cell Res. 315, 2791-2801. doi: 10.1016/j.yexcr.2009. 06.023

Novichkova, S., Egorov, S., and Daraselia, N. (2003a). MedScan, a natural language processing engine for MEDLINE abstracts. Bioinformatics 19, 1699-1706. doi: 10.1093/bioinformatics/btg207

Novichkova, S., Egorov, S., and Daraselia, N. (2003b). MedScan, a natural language processing engine for MEDLINE abstracts. Bioinformatics 19, 1699-1706. doi: 10.1093/bioinformatics/btg207

Nunomura, A., Perry, G., Aliev, G., Hirai, K., Takeda, A., Balraj, E. K., et al. (2001). Oxidative damage is the earliest event in Alzheimer disease. J. Neuropathol. Exp. Neurol. 60, 759-767.

Olanow, C. W., and McNaught, K. S. (2006). Ubiquitin-proteasome system and Parkinson's disease. Mov. Disord. 21, 1806-1823. doi: 10.1002/mds.21013

Oresic, M., Hyotylainen, T., Herukka, S.-K., Sysi-Aho, M., Mattila, I., SeppananLaakso, T., et al. (2011). Metabolome in progression to Alzheimer's disease. Transl. Psychiatry 1:e57. doi: 10.1038/tp.2011.55

Paquet, D., Bhat, R., Sydow, A., Mandelkow, E. M., Berg, S., Hellberg, S., et al. (2009). A zebrafish model of tauopathy allows in vivo imaging of neuronal cell death and drug evaluation. J. Clin. Invest. 119, 1382-1395. doi: 10.1172/JCI37537

Pittman, A. M., Fung, H. C., and De Silva, R. (2006). Untangling the tau gene association with neurodegenerative disorders. Hum. Mol. Genet. 15(Spec No. 2), R188-R195. doi: 10.1093/hmg/ddl190

Pluta, R. (2005). Pathological opening of the blood-brain barrier to horseradish peroxidase and amyloid precursor protein following ischemia-reperfusion brain injury. Chemotherapy 51, 223-226. doi: 10.1159/000086924

Pujic, Z., Omori, Y., Tsujikawa, M., Thisse, B., Thisse, C., and Malicki, J. (2006) Reverse genetic analysis of neurogenesis in the zebrafish retina. Dev. Biol. 293 330-347. doi: 10.1016/j.ydbio.2005.12.056

Rademakers, R., and Rovelet-Lecrux, A. (2009). Recent insights into the molecular genetics of dementia. Trends Neurosci. 32, 451-461. doi: 10.1016/j.tins.2009.05.005

Raymond, P. A., Barthel, L. K., Bernardos, R. L., and Perkowski, J. J. (2006). Molecular characterization of retinal stem cells and their niches in adult zebrafish. BMC Dev. Biol. 6:36. doi: 10.1186/1471-213X-6-36

Regeur, L., Jensen, G. B., Pakkenberg, H., Evans, S. M., and Pakkenberg, B. (1994). No global neocortical nerve cell loss in brains from patients with senile dementia of Alzheimer's type. Neurobiol. Aging 15, 347-352. doi: 10.1016/0197-4580(94)90030-2

Roberds, S. L., Anderson, J., Basi, G., Bienkowski, M. J., Branstetter, D. G., Chen, K. S., et al. (2001). BACE knockout mice are healthy despite lacking the primary beta-secretase activity in brain: implications for Alzheimer's disease therapeutics. Hum. Mol. Genet. 10, 1317-1324. doi: 10.1093/hmg/10. 12.1317

Ross, C. A., and Poirier, M. A. (2004). Protein aggregation and neurodegenerative disease. Nat. Med. 10(Suppl.), S10-S17. doi: 10.1038/nm1066 
Santana, S., Rico, E. P., and Burgos, J. S. (2012). Can zebrafish be used as animal model to study Alzheimer's disease? Am. J. Neurodegener. Dis. 1, 32-48.

Schmid, B., and Haass, C. (2013). Genomic editing opens new avenues for zebrafish as a model for neurodegeneration. J. Neurochem. 127, 461-470. doi: 10.1111/jnc. 12460

Selfridge, J. E., E, L., Lu, J., and Swerdlow, R. H. (2012). Role of mitochondrial homeostasis and dynamics in Alzheimer's disease. Neurobiol. Dis. 51, 3-12. doi 10.1016/j.nbd.2011.12.057

Shen, J., Bronson, R. T., Chen, D. F., Xia, W., Selkoe, D. J., and Tonegawa, S. (1997). Skeletal and CNS defects in Presenilin-1-deficient mice. Cell 89, 629-639. doi: 10.1016/S0092-8674(00)80244-5

Song, P., and Pimplikar, S. W. (2012). Knockdown of amyloid precursor protein in zebrafish causes defects in motor axon outgrowth. PLoS ONE 7:e34209. doi: 10.1371/journal.pone.0034209

Stone, J. (2008). What initiates the formation of senile plaques? The origin of Alzheimer-like dementias in capillary haemorrhages. Med. Hypotheses 71, 347359. doi: 10.1016/j.mehy.2008.04.007

Strausberg, R. L., Feingold, E. A., Grouse, L. H., Derge, J. G., Klausner, R. D., Collins, F. S., et al. (2002). Generation and initial analysis of more than 15,000 full-length human and mouse cDNA sequences. Proc. Natl. Acad. Sci. U.S.A. 99, 16899-16903. doi: 10.1073/pnas.242603899

Sundvik, M., Chen, Y. C., and Panula, P. (2013). Presenilin1 regulates histamine neuron development and behavior in zebrafish, danio rerio. J. Neurosci. 33, 15891597. doi: 10.1523/JNEUROSCI.1802-12.2013

Tingaud-Sequeira, A., Forgue, J., Andre, M., and Babin, P. J. (2006). Epidermal transient down-regulation of retinol-binding protein 4 and mirror expression of apolipoprotein $\mathrm{Eb}$ and estrogen receptor 2a during zebrafish fin and scale development. Dev. Dyn. 235, 3071-3079. doi: 10.1002/dvdy. 20921

Tseng, W. E., Chen, C. M., Chen, Y. C., Yi, Z., Tan, E. K., and Wu, Y. R. (2013). Genetic Variations of GAK in two Chinese Parkinson's disease populations: a case-control study. PLoS ONE 8:e67506. doi: 10.1371/journal.pone.0067506

van Bebber, F., Hruscha, A., Willem, M., Schmid, B., and Haass, C. (2013). Loss of Bace2 in zebrafish affects melanocyte migration and is distinct from Bace1 knock out phenotypes. J. Neurochem. 127, 471-481. doi: 10.1111/jnc.12198

van Tijn, P., Kamphuis, W., Marlatt, M. W., Hol, E. M., and Lucassen, P. J. (2011). Presenilin mouse and zebrafish models for dementia: focus on neurogenesis. Prog. Neurobiol. 93, 149-164. doi: 10.1016/j.pneurobio.2010.10.008

Verdile, G., Fuller, S., Atwood, C. S., Laws, S. M., Gandy, S. E., and Martins, R. N. (2004). The role of beta amyloid in Alzheimer's disease: still a cause of everything or the only one who got caught? Pharmacol. Res. 50, 397-409. doi: 10.1016/j.phrs.2003.12.028

Veth, K. N., Willer, J. R., Collery, R. F., Gray, M. P., Willer, G. B., Wagner, D. S., et al. (2011). Mutations in zebrafish $\operatorname{lrp} 2$ result in adult-onset ocular pathogenesis that models myopia and other risk factors for glaucoma. PLoS Genet. 7:e1001310. doi: 10.1371/journal.pgen.1001310

Voisin, T., and Vellas, B. (2009). Diagnosis and treatment of patients with severe Alzheimer's disease. Drugs Aging 26, 135-144. doi: 10.2165/0002512-20092602000005
West, M. J., Coleman, P. D., Flood, D. G., and Troncoso, J. C. (1994) Differences in the pattern of hippocampal neuronal loss in normal ageing and Alzheimer's disease. Lancet 344, 769-772. doi: 10.1016/S0140-6736(94) 92338-8

Willem, M., Garratt, A. N., Novak, B., Citron, M., Kaufmann, S., Rittger, A., et al. (2006). Control of peripheral nerve myelination by the beta-secretase BACE1. Science 314, 664-666. doi: 10.1126/science.1132341

Wilson, L., and Lardelli, M. (2013). The development of an in vivo gamma-secretase assay using zebrafish embryos. J. Alzheimers Dis. 36, 521-534. doi: 10.3233/JAD130332

Wong, P. C., Zheng, H., Chen, H., Becher, M. W., Sirinathsinghji, D. J., Trumbauer, M. E., et al. (1997). Presenilin 1 is required for Notch1 and DII1 expression in the paraxial mesoderm. Nature 387, 288-292. doi: 10.1038/387288a0

Woods, I. G., Wilson, C., Friedlander, B., Chang, P., Reyes, D. K., Nix, R., et al. (2005). The zebrafish gene map defines ancestral vertebrate chromosomes. Genome Res. 15, 1307-1314. doi: 10.1101/gr.4134305

World Alzheimer report. (2010). "Alzheimer's Disease International 2010," in World Alzheimer Report 2010: The Global Impact of Dementia, eds A. Wimo and M. Prince (London: Alzheimer's Disease International).

Yang, T., Arslanova, D., Xu, X., Li, Y. M., and Xia, W. (2010). In vivo manifestation of notch related phenotypes in zebrafish treated with Alzheimer's amyloid reducing gamma-secretase inhibitors. J. Neurochem. 113, 1200-1209. doi: 10.1111/j.14714159.2010.06681.x

Zetterberg, H., Mortberg, E., Song, L., Chang, L., Provuncher, G. K., Patel, P. P., et al. (2011). Hypoxia due to cardiac arrest induces a time-dependent increase in serum amyloid beta levels in humans. PLOS ONE 6:e28263. doi: 10.1371/journal.pone.0028263

Zhang, X., Garbett, K., Veeraraghavalu, K., Wilburn, B., Gilmore, R., Mirnics, K., et al. (2012). A role for presenilins in autophagy revisited: normal acidification of lysosomes in cells lacking PSEN1 and PSEN2. J. Neurosci. 32, 8633-8648. doi: 10.1523/JNEUROSCI.0556-12.2012

Zhang, X., and Le, W. (2010). Pathological role of hypoxia in Alzheimer's disease. Exp. Neurol. 223, 299-303. doi: 10.1016/j.expneurol.2009.07.033

Conflict of Interest Statement: The authors declare that the research was conducted in the absence of any commercial or financial relationships that could be construed as a potential conflict of interest.

Received: 07 March 2014; accepted: 06 June 2014; published online: 30 June 2014. Citation: Newman M, Ebrahimie E and Lardelli M (2014) Using the zebrafish model for Alzheimer's disease research. Front. Genet. 5:189. doi: 10.3389/fgene.2014.00189 This article was submitted to Genetics of Aging, a section of the journal Frontiers in Genetics.

Copyright (c) 2014 Newman, Ebrahimie and Lardelli. This is an open-access article distributed under the terms of the Creative Commons Attribution License (CC BY). The use, distribution or reproduction in other forums is permitted, provided the original author(s) or licensor are credited and that the original publication in this journal is cited, in accordance with accepted academic practice. No use, distribution or reproduction is permitted which does not comply with these terms. 